\title{
Frühgeburtlichkeit und langfristige Folgen bis ins Schulalter
}

\author{
Implikationen für die Nachsorge und Schule
}

\author{
Britta Maria Hüning ${ }^{1}$ und Julia Jäkel²
}

${ }^{1}$ Neonatologie, Pädiatrische Intensivmedizin und Neuropädiatrie, Klinik für Kinderheilkunde I, Universitätsklinikum Essen

${ }^{2}$ Department of Child and Family Studies, Department of Psychology, University of Tennessee, Knoxville, USA

\begin{abstract}
Zusammenfassung: Theoretischer Hintergrund: Frühgeborene (FG) haben ein erhöhtes langfristiges Entwicklungsrisiko. Dennoch gibt es in Deutschland kein konzertiertes Vorgehen zur Nachsorge bis ins Schulalter. Die heutigen Erkenntnisse zu Entwicklungsstörungen sind Grundlage einer qualifizierten Förderung. Fragestellung: Wie hoch sind Schulrückstellungsraten bei FG? Wie wird den schulischen Bedürfnissen FG Rechnung getragen? Methode: Evaluation der Schulrückstellung in einer aktuellen Kohorte sehr kleiner FG und qualitative Befragung von Lehrer_innen. Ergebnisse: Das Risiko für Schulrückstellungen ist bei FG erhöht. Lehrer_innen haben ein limitiertes Wissen zu Bedürfnissen FG und gleichzeitig hilfreiche Vorschläge für spezifische Förderung im Unterricht. Diskussion und Schlussfolgerung: Langfristige entwicklungsneurologische Nachsorge für FG ist dringend empfohlen, um potenzielle Probleme früh zu identifizieren, Interventionen zu initiieren und eine optimale Entfaltung des Entwicklungspotentials zu fördern.
\end{abstract}

Schlüsselwörter: Frühgeborene, langfristige Entwicklung, Nachsorge, Schulrückstellung, Förderung Schule

\section{Preterm Birth and Long-Term Consequences up to School Age. Implications for Follow-Up Services and Schooling}

Abstract: Theoretical Background: Survival rates of very immature preterm infants born before $28+0$ weeks' gestation have increased in the past few decades. However, a birth earlier than full term $(<37+0$ weeks' gestation) is associated with a gradual increase in long-term developmental risk. Follow-up studies have documented a substantial personal and financial burden for individuals, their families, as well as health and education systems. This highlights the need for structured long-term care and follow-up services for premature infants. In Germany, there are no standardized follow-up protocols for preterm infants, apart from one evaluation of "late outcome quality" at 24 months corrected age for preterm infants with a birth weight under 1,500 g (Joint Federal Committee - Gemeinsamer Bundesausschuss, GBA). Parents and teachers often expect preterm children's development to catch up with peers by the time they enter school, but the opposite is the case: Many preterm children struggle with attention and working memory and they often have difficulties in learning. Furthermore, studies have shown that education professionals are not prepared to support preterm children's specific needs in the classroom. Objective: We aim to present the current knowledge on the long-term development of preterm infants as a basis for evidencebased follow-up policies. Special attention is paid to school readiness, delayed school entry, and the specific educational needs of preterm children. Method: Data from two sources are presented. The first considered delayed entry into formal schooling, evaluated in a recent cohort of very preterm children compared with term controls, enrolled in school between 2017 and 2019 in an urban region in Germany. In the second, qualitative survey data are included and represent US teachers' suggestions on how they would specifically support preterm children in the classroom. Results: Delayed school entry: Very preterm children had a delayed school entry more often than their term-born peers. While school entry was delayed in $12.5 \%$ of preterm infants, this only happened in $1.7 \%$ of term-comparison children (Fisher's exact probability test $p=.029$ ). Qualitative responses provided insight into how teachers could support preterm children's academic performance, attention, peer relationships, and emotional regulation in the classroom. Discussion and Conclusion: Considering specific needs of preterm children, neurodevelopmental follow-up is strongly recommended to medical and educational professionals and policy-makers. Multidisciplinary cooperation between different professionals and interest groups, with a focus on strength-based approaches, is essential. Parents and preterm infants themselves need to be included in all steps of the process. Early diagnosis and intervention may improve future outcomes and therefore support optimal development and quality of life throughout childhood and beyond.

Keywords: preterm infants, follow-up, delayed school entry, educational support 


\section{Frühgeburt - Häufigkeit, Definition und Mortalität}

Eine zu frühe Geburt stellt weltweit eines der schwerwiegendsten Entwicklungsrisiken für Neugeborene dar. Im Jahr 2018 wurden in Deutschland 64417 (8,4\%) Kinder vor der vollendeten 37. Schwangerschaftswoche (SSW) zu früh geboren (Institut für Qualitätssicherung und Transparenz im Gesundheitswesen, 2019). Neugeborene mit einem Gestationsalter von weniger als 259 Tagen bzw. $<37+0$ Schwangerschaftswochen (SSW), gerechnet ab dem 1 . Tag der letzten Periode, werden als Frühgeborene bezeichnet. Frühgeborene werden entsprechend ihres Gestationsalters (errechneten SSW) bzw. ihres Gewichtes bei Geburt in graduelle (Risiko-) Gruppen eingeteilt (s. Tab. 1).

Tabelle 1. Die Einteilung Frühgeborener erfolgt nach dem Gestationsalter bzw. dem Gewicht in Gramm (g) bei Geburt

\begin{tabular}{|c|c|}
\hline \multicolumn{2}{|c|}{ Einteilung nach dem Gestationsalter } \\
\hline \multicolumn{2}{|c|}{ Geburt vor der vollendeten } \\
\hline 28. SSW & Extrem unreife Frühgeburt \\
\hline 32. SSW & Sehr unreife Frühgeburt \\
\hline 34. SSW & Moderate Frühgeburt \\
\hline 36. SSW & Späte Frühgeburt \\
\hline \multicolumn{2}{|c|}{ Einteilung nach dem Geburtsgewicht } \\
\hline$<1000 \mathrm{~g}$ & Extremely low birth weight (ELBW) \\
\hline$<1500 \mathrm{~g}$ & Very low birth weight (VLBW) \\
\hline$<2500 \mathrm{~g}$ & Low birth weight (LBW) \\
\hline 10. - 90. Perzentile & Eutrophes Frühgeborenes \\
\hline$<10$. Perzentile & Hypotrophes Frühgeborenes \\
\hline$>$ 90. Perzentile & Hypertrophes Frühgeborenes \\
\hline
\end{tabular}

Die Überlebenswahrscheinlichkeit, v.a. der extrem unreifen Frühgeborenen ( $<28 \mathrm{SSW}$ ) hat sich in den vergangenen Jahrzehnten durch Fortschritte in der Neonatalmedizin deutlich verbessert (Pierrat et al., 2017, Santhakumaran et al., 2018). Das Risiko für neonatale Komplikationen, Entwicklungsprobleme und langfristige Morbidität bleibt jedoch durch die gestiegene Überlebensrate dieser extrem unreifen Kinder auf hohem Niveau stabil (Moore et al., 2012; Stoll et al., 2015; Patel, 2016; Helenius et al., 2017). Die neuere Literatur liefert uns zudem immer mehr Daten zu moderaten und späten Frühgeborenen. Sie machen weltweit den größten Anteil (80 \%) der neonatalen Risikokinder aus und weisen ebenfalls eine graduell höhere Rate an Entwicklungsstörungen auf als reifgeborene Kinder (Woythaler, 2019; Cheong et al., 2020).

Die Qualität der neonatologischen Versorgung misst sich daher nicht länger nur an der Überlebensrate Früh- geborener, sondern vielmehr an den langfristigen Lebenschancen und der Lebensqualität dieser Kinder. Auswirkungen der Frühgeburt sind auch in zukünftigen Generationen zu erwarten. Aus diesem Grund muss dringend eine flächendeckende langfristige Nachsorge eingeführt, und wirksame Interventionsansätze zur Verbesserung der neurokognitiven Entwicklung evaluiert werden.

Ziel unseres Beitrags ist es, die heutigen Erkenntnisse zu Auswirkungen einer Frühgeburt auf die physische und psychische Gesundheit und zur langfristigen Entwicklung Frühgeborener als Grundlage einer qualifizierten Langzeitbetreuung darzustellen. Die rechtlichen Vorgaben der Nachsorge werden ebenso dargestellt, wie die relevanten Entwicklungsdimensionen, die Gegenstand einer strukturierten und standardisierten Nachsorge sein sollten. Besondere Aufmerksamkeit widmen wir im letzten Teil unseres Artikels dem Schuleintritt und den schulischen Bedürfnissen Frühgeborener. Hierzu werden eigene Daten vorgestellt.

\section{Frühgeburt und langfristige Folgen}

Eine Vielzahl von Studien hat Entwicklungsprobleme nach einer Frühgeburt bis ins Erwachsenenalter dokumentiert. Dazu zählen unter anderem Aufmerksamkeitsprobleme, niedrige Bildungsabschlüsse, geringe finanzielle Selbständigkeit, und eingeschränkte Lebensqualität (Moster et al., 2008; Hack, 2009; D'Onofrio et al., 2013; Darlow et al., 2013; Heinonen et al.; 2013; Johnson et al., 2014; Bilgin et al., 2018). Die Frühgeburtlichkeit stellt damit nicht nur die Familie und das Kind vor große Herausforderungen, sondern belastet auch die Gesundheitsund Bildungssysteme (Petrou et al., 2009; Khan et al., 2015).

Bei der Betrachtung der langfristigen Entwicklung Frühgeborener müssen zunächst einige Faktoren beachtet werden: 1.) Die Behandlungsergebnisse ehemaliger Frühgeborener, die heute im mittleren Erwachsenenalter sind, lassen sich nicht 1:1 auf heutige Frühgeborene anwenden. In Westeuropa werden Frühgeborene gegenwärtig überwiegend in spezialisierten Zentren geboren, erhalten nicht-invasive Atemunterstützung und schonende Behandlungsverfahren. Damit sind sie im Vergleich zu in früheren Epochen geborenen Frühgeborenen deutlich geringeren Risiken für Hirnschädigungen ausgesetzt. Zudem erlangen Maßnahmen zum Schutz des unreifen Gehirns und familienzentrierte Betreuungsansätze eine immer größere Bedeutung mit dem Ziel, die neurologische Entwicklung weiter zu verbessern (Huening et al., 2012; DeMaster et al., 2019; van Bel et al., 2019). 
Die Versorgung von Früh- und Neugeborenen ist in Deutschland stark dezentralisiert mit mehr als 300 Zentren. Es existiert kein bundesweit verbindliches Register zur Erfassung der langfristigen Entwicklung Frühgeborener. Die Kenntnisse zum langfristigen Outcome werden daher überwiegend aus internationalen, populationsbasierten Studien bezogen. (Moore et al., 2012; Ancel et al., 2015; Saigal et al., 2016; Serenius et al., 2016). Im Rahmen von einzelnen Studien wie z. B. der Bayerischen Entwicklungsstudie (BESt) (Jaekel, Baumann, et al., 2019; Jaekel, Sorg, et al., 2019) sind sehr gut dokumentierte deutsche Daten bis ins Erwachsenenalter, verfügbar. Seit 2009 existiert zudem der Forschungsverbund des German Neonatal Network (GNN) an dem mehr als 50 größere Zentren beteiligt sind (Herting et al., 2020; Humberg et al., 2020).

Die Zahl von Follow-up Studien und „Individual Patient Meta-Analyses (IPD)“, zum Beispiel im Rahmen der Adults born Preterm International Collaboration (APIC) oder des von der EU geförderten Forschungsprojekts Research on European Children and Adults Born Preterm (RECAP), bis ins Erwachsenenalter wächst. Diese machen deutlich, dass nicht nur das Gestationsalter bei der Geburt, sondern auch die Ursachen, die zur Frühgeburt geführt haben, einen substantiellen Einfluss auf die langfristige physische und psychische Gesundheit und die soziale Integration haben können (Singer, 2006; Saigal et al., 2016; Raju et al., 2017; Mendonca et al., 2019).

\section{Auswirkungen der Frühgeburtlichkeit auf die physische und psychische Gesundheit}

Für die Entwicklung Frühgeborener gelten einige universelle Grundsätze, die in der Unreife der Organsysteme bzw. der Unterbrechung der normativen pränatalen Reifungsprozesse begründet zu sein scheinen: Je früher ein Kind zur Welt kommt und je limitierter das intrauterine Wachstum war, desto höher ist zumeist das Risiko für eine langfristige Morbidität (Singer, 2006; Platt, 2014).

Sehr unreife Frühgeborene, insbesondere jene mit chronischer Lungenerkrankung (Bronchopulmonale Dysplasie), haben in den ersten Lebensjahren ein erhöhtes Risiko für wiederkehrende Infektionen mit Verengung der oberen Atemwege (spastische Bronchitiden). Etwa ein Drittel der Kinder leidet unter Asthma (Korvenranta et al., 2009; Greenough, 2013). Die reduzierte Lungenfunktion persistiert bis ins Erwachsenenalter und führt zu geringerer aerober Belastbarkeit (Lovering et al., 2014). Frühgeborene, auch jene mit höherem Gestationsalter bei Geburt (32-36 SSW), haben zudem ein erhöhtes Risiko für kardiovaskuläre und metabolische Erkrankungen (Bluthochdruck und Typ-II Diabetes mellitus). Als primärer
Mechanismus wird die frühe Stressexposition benannt, die das endokrine und metabolische System über die Hypothalamus-Hypophysen-Nebennieren-Achse lebenslang beeinflussen kann. (Parkinson et al., 2013; Hovi et al., 2016; Morrison et al., 2016; Markopoulou et al., 2019; Cheong \& Doyle, 2020).

Für das Gehirn werden die Folgen einer Frühgeburt schon lange intensiv untersucht. Galt die Aufmerksamkeit zunächst v.a. den Folgen einer Hirnblutung oder einer Sauerstoffunterversorgung, die zu motorischen Lähmungen im Sinne einer Zerebralparese führen können, so ist durch einen Rückgang dieser schwerwiegenden Komplikationen (van Haastert et al., 2011) v.a. die kognitive Entwicklung in den Fokus der Aufmerksamkeit gerückt. Kognitive Defizite ohne gravierende motorische Behinderung stellen gegenwärtig die häufigste Form entwicklungsneurologischer Probleme dar (Volpe, 2009). Nach Rückgang der gravierenden Hirnschädigungen dominieren heute mikroskopische Auffälligkeiten der weißen Substanz (Schädigung der Nervenfasern und geringere Integrität der Myelinscheiden), die in Summe mit einer Erweiterung der inneren und äußeren Liquorräume und einer variablen Beteiligung der kortikalen und tiefen grauen Substanz (Nervenzellen), sowie des Kleinhirns, einhergehen und zu einer geringeren Komplexität zerebraler Netzwerke (weniger gut organisierte Nervenfaserverbindungen zwischen verschiedenen Hirnregionen) führen (Woodward et al., 2006; Volpe, 2011; Rogers et al., 2018).

Abhängig von Schwangerschaftsdauer und der Schwere des neonatalen Risikos ist diese Reorganisation kortikaler Strukturen noch in der Kindheit, Jugend und selbst im Erwachsenenalter nachweisbar (Peterson, 2003; Nosarti et al., 2010; Bäuml et al., 2014). Diese Veränderungen der Hirnentwicklung hängen prospektiv mit akademischem Erfolg zusammen, da sie auf funktioneller Ebene zu Aufmerksamkeits- und Arbeitsgedächtnisdefiziten (Woodward et al., 2012) sowie langsamerer Verarbeitungsgeschwindigkeit führen (Mulder et al., 2010).

Bei bis zu 50 \% der extrem kleinen Frühgeborenen bestehen im Schulalter Einschränkungen der allgemeinen Intelligenz, in sprachlichen Bereichen, im mathematischen Denken, in exekutiven Funktionen, assoziativen Lernvorgängen, und im sozial-emotionalen Verhalten (Marlow et al., 2005; Lohaugen et al., 2010; Johnson et al., 2016; Raju et al., 2017; Tran et al., 2017; Twilhaar et al., 2018). Moderate und späte Frühgeborene haben ebenso ein graduell erhöhtes Risiko für schulischen Misserfolg (MacKay et al., 2010; Boyle, 2012; Lipkind et al., 2012; Quigley et al., 2012; Poulsen et al., 2013). Die sprachlichen Schwierigkeiten Frühgeborener treten mit zunehmendem Alter deutlicher zutage, v. a. in komplexen sprachlichen Funktionen (Bildung von Haupt- und Ne- 
bensätzen, relationale Begriffe, Verben) (van Noort-van der Spek et al., 2012) und wirken sich auf das schulische Lernen ebenso wie auf soziale Interaktionen aus (St Clair et al., 2011).

Im Bereich der sozialen Beziehungen zeigen Frühgeborene häufiger Persönlichkeitsmerkmale wie Ängstlichkeit und Schüchternheit. So sind sie häufig unsicherer und zurückhaltend im sozialen Kontakt (Reyes, Jaekel, Heuser, et al., 2019; Reyes, Jaekel \& Wolke, 2019), schließen weniger Freundschaften, verbringen weniger Zeit mit Freunden und können häufiger Opfer von Mobbing werden (Wolke et al., 2015; Heuser et al., 2018). Frühgeborene leiden hingegen nicht grundsätzlich häufiger an Angstoder affektiven Störungen (Jaekel et al., 2018). Aufmerksamkeitsdefizit-/Hyperaktivitäts-Störung und Störungen aus dem Autismusspektrum treten jedoch bei sehr unreifen Frühgeborenen häufiger auf als bei reifgeborenen Kindern (Johnson \& Marlow, 2011).

Das Risiko für geringere akademische und sozial-emotionale Kompetenzen beeinträchtigen die Lebenschancen erheblich, was sich in niedrigeren Schulabschlüssen, geringeren Durchschnittseinkommen und weniger finanzieller Unabhängigkeit widerspiegelt (Moster et al., 2008; Raju et al., 2017; Bilgin et al., 2018; Jaekel, Baumann et al., 2019; Wolke et al., 2019).

Diese Erkenntnisse machen die Notwendigkeit einer strukturierten Langzeitbetreuung Frühgeborener und ihrer Familien deutlich. Nachsorgeangebote sollten dabei verschiedene Bereiche berücksichtigen: Wachstum, Ernährung, physische Gesundheit, sensorische Funktionen, die motorische, kognitive, sprachliche und sozioemotionale Entwicklung. Auffälligkeiten sollen so früh wie möglich identifiziert und individuelle Therapie- und Fördermaßnahmen eingeleitet werden. Dies erfordert die $\mathrm{Zu}-$ sammenarbeit in einem multi-disziplinären Team und die Kooperation mit Therapeut_innen und Lehrern, um doppelte Diagnostik ebenso wie Versorgungslücken zu vermeiden.

\section{Nachsorge in Deutschland - rechtsverbindliche Vorgaben}

Da strukturierte und langfristig angelegte Nachuntersuchungsprogramme in Deutschland fehlen, fallen viele Kinder erst im Schulalter mit Problemen auf (LenzenSchulte, 2018). Insbesondere für Frühgeborene mit höheren Gestationsaltern bestehen weder etablierte Protokolle, noch erlauben personelle wie finanzielle Ressourcen eine flächendeckende, standardisierte Nachsorge.
Der Gemeinsame Bundesausschuss (G-BA) sieht seit Januar 2006 Folgeuntersuchungen von Frühgeborenen mit einem Geburtsgewicht von $<1500 \mathrm{~g}$ im korrigierten Alter von 24 Monaten vor. Perinatalzentren der beiden höchsten Versorgungsstufen müssen diese „späte Ergebnisqualität" ihrer Versorgung veröffentlichen, ( $\$ 4 \mathrm{Ab}$ satz 1) (G-BA, 2017). Diese Daten sollen ein bundesweites Benchmarking neonatologischer Versorgungsqualität ermöglichen, problematisch ist jedoch, dass als Kriterium für Folgeuntersuchungen alleinig das Geburtsgewicht, nicht das tatsächliche Gestationsalter bei Geburt zu Grunde gelegt wird, da v.a. die mangelnde Organreife ursächlich für viele Erkrankungen Frühgeborener ist.

In der späten Ergebnisqualität (G-BA) kommen folgende Variablen zur Darstellung: Vollständigkeit der Folgeuntersuchungen, Anzahl blinder oder schwerhöriger Kinder, schwere motorische Funktionsstörungen (Zerebralparesen) und schwere mentale Retardierung (z. B. Motor Developmental Index (MDI) im Bayley Scales of Infant Development (BSID) <55), sowie die Anzahl von Kindern, die keine oder eine nur geringfügige mentale Retardierung aufweisen (z. B. MDI > $70 \mathrm{im}$ BSID).

Diese Ergebnisse können aus zahlreichen Gründen aber nur ein unvollständiges Bild über die Folgen der Frühgeburtlichkeit liefern: 1.) Die „späte Ergebnisqualität" erfasst nur die schwerwiegenden Behinderungen (sensorische Störungen und Zerebralparesen). Diese können zu diesem Zeitpunkt mit hoher Sicherheit diagnostiziert werden, ebenso schwerwiegende mentale Beeinträchtigungen, die sich vom 2. Lebensjahr über die Kindheit und Jugend bis ins Erwachsenenalter recht stabil zeigen (Breeman et al., 2015; Linsell et al., 2018). Mildere motorische und kognitive Funktionseinschränkungen und Lernstörungen hingegen werden erst dann evident, wenn ein Lebensalter erreicht ist, in dem diese Anforderungen zunehmende Bedeutung erlangen. 2.) Die Mehrheit der sehr kleinen Frühgeborenen ist jedoch von milden kognitiven Defiziten ohne schwerwiegende motorische Auffälligkeiten betroffen. Diese können sich verbessern oder verschlechtern und sollten daher wiederholt überprüft werden, um eine angemessene Förderung des Kindes zu ermöglichen (Orton et al., 2009; Spittle et al., 2015).

Der G-BA schreibt für die „späte Ergebnisqualität“ weder eine vollständige standardisierte Testung aller Entwicklungsbereiche mit korrigiert 24 Monaten noch weitere Untersuchungen, z. B. im Vorschulalter, vor.

Verschiedene Experten, Fachgesellschaften und Elternverbände empfehlen daher eine Nachsorge bis ins Schulalter (Arbeitsgemeinschaft der Wissenschaftlichen Medizinischen Fachgesellschaften (AWMF), 2018; European Foundation for the care of newborn infants (EFCNI), 2018). Die eingesetzten Testinstrumente und Untersuchungsverfahren sollten eine ausreichende Validität 
nachweisen, von Untersuchern mit entsprechender Qualifikation und Erfahrung in der Anwendung durchgeführt werden, und die Muttersprache der Kinder berücksichtigen (Jaekel, 2020; Kajantie, 2020).

\section{Relevante Dimensionen der entwicklungsneurologischen Nachsorge im Laufe der Kindheit}

In seiner Entwicklung erwirbt das Kind Kompetenzen und Fähigkeiten, die von der komplexen Interaktion aus biologischen Faktoren und Umweltfaktoren abhängig sind. In sensiblen Phasen der Reorganisation von Nervenfaserverbindungen, die mit dem Erwerb spezifischer Fähigkeiten einhergehen, haben Umwelterfahrungen einen besonders starken Einfluss (Pluess, 2015). Maßgeblich werden diese Erfahrungen in den ersten Lebensjahren durch die häusliche Umgebung und die reziproke Interaktion mit den Eltern geprägt (Feldman, 2015). Das Fürsorgeund Erziehungsverhalten der Eltern hat großen Einfluss auf die Entwicklung des Kindes (Jaekel \& Wolke, 2014). Grundlegende Fähigkeiten des Kindes bilden die Basis für das Erlernen höherer, komplexer Funktionen, die mit der Reifung des präfrontalen Kortex einhergehen (Hodel, 2018).

Das Spektrum möglicher Folgekomplikationen Frühgeborener wandelt sich im Laufe der Kindheit und neuen Problemen muss in der Nachsorge Rechnung getragen werden. Für die verschiedenen Altersbereiche werden daher im Folgenden die für die Nachsorge relevanten Dimensionen der Entwicklung dargestellt (s. Tab. 2).

Im ersten Lebensjahr stehen das Wachstum, die medizinischen Komplikationen, wie z.B. rezidivierende Infekte, sensorische Störungen und die motorische Entwicklung des Kindes im Vordergrund (Huening et al., 2018). Die Förderung des Aufholwachstums kann das neurologische Ergebnis Frühgeborener günstig beeinflussen (Stenson et al., 2016). Jaekel, Sorg et al. (2019) konnten zudem zeigen, dass das Kopfwachstum in der Kindheit ein gutes prognostisches Maß für die Intelligenz im Alter von 6 bis 26 Jahren darstellt (Jaekel, Sorg et al., 2019).

Hörstörungen treten bei 2-4\% der sehr kleinen Frühgeborenen auf und sollten vor dem 6 . Lebensmonat diagnostiziert werden, um Therapiemaßnahmen einzuleiten und die sprachliche Entwicklung zu fördern (Pimperton et al., 2016; Hirvonen et al., 2018). Das Sehvermögen kann insbesondere bei Kindern nach einer zerebralen Schädigung beeinträchtigt sein. Daher sollten Refraktionsfehler und ein Strabismus (Schielen) zum Ende des ersten Lebensjahres überprüft werden.

In der Früherkennung einer zentralen Bewegungsstörung hat sich neben bildgebenden Verfahren die Analyse altersspezifischer Bewegungsmuster (General Movements Assessment) in der Neonatalzeit und in den ersten Lebensmonaten etabliert (Einspieler et al., 2004; Bosanquet et al., 2013). Im korrigierten Alter von 3 bis 5 Monaten zeigte sich die Abwesenheit s.g. "Fidgety Movements“ als prädiktiv für die Entwicklung einer spastischen Zerebralparese (Sensitivität: 98\%, Spezifität: 95\%), (Bosanquet et al., 2013; Kwong et al., 2018; Einspieler et al., 2019).

Dieser einfach durchzuführenden, nicht-invasiven Beobachtung und Klassifikation der Spontanmotorik kommt nicht nur in der Beratung der Eltern eine große Bedeutung zu, sondern auch in der Planung frühzeitiger Interventionen mit dem Ziel Komplikationen (Kontrakturen, Deformitäten) zu reduzieren.

Ein weiteres Augenmerk sollte auf die Eltern gerichtet sein, da deren psychische Gesundheit Einfluss auf die Entwicklung des Kindes hat (Feldman et al., 2009). Eine Frühgeburt kann zu Depressionen und Posttraumatischen Belastungsstörungen führen (Vigod et al., 2010, Pace et al., 2016). Daher sind Screeningtools für Depressionen im ersten Lebensjahr des Kindes und die psychotherapeutische Versorgung ratsam.

Eltern Frühgeborener sind oft zusätzlichen Stressbelastungen ausgesetzt (Wolke et al., 2017), während Frühgeborene besonders von einem sensitiven und responsiven Verhalten profitieren (Bilgin et al., 2016). Die Zeit der Schwangerschaft und das erste Lebensjahr stellen einen optimalen Zeitpunkt für die Unterstützung von Eltern in der Übernahme ihrer neuen Rolle und der Bindung zum Kind dar (s.g. „two generation approach") (Shonkoff et al., 2013), jedoch sollte auch die langfristige Unterstützung der Eltern, insbesondere während kritischer Entwicklungsfenster wie Schuleintritt oder Adoleszenz, nicht vernachlässigt werden (Jaekel, 2016).

Im zweiten Lebensjahr sollten Therapiemaßnahmen (Behandlung bei Ess- und Fütterstörungen, sinnesspezifische Förderung, spezifische Physio- und Ergotherapie, Versorgung mit Hilfsmitteln, etc.) eingeleitet sein und Förderkonzepte (Frühförderung als familienorientierte Komplexleistung bei drohender Behinderung, pädagogische Familienhilfe, etc.) bedürfnisorientiert angepasst werden.

Viele Zentren in Deutschland bieten entsprechend des G-BA-Beschlusses eine Folgeuntersuchung mit korrigiert 24 Monaten an und verwenden den BSID II oder III zur Erhebung des globalen Entwicklungsstandes, bzw. einzelner Bereiche.

Im Vorschulalter rückt zunehmend die kognitive Entwicklung in den Vordergrund. Exekutive Funktionen entwickeln sich abhängig von Reifungsprozessen des präfrontalen Kortex rasch in diesem Alter und sind entscheidender für den späteren Schulerfolg als der Intelligenz- 


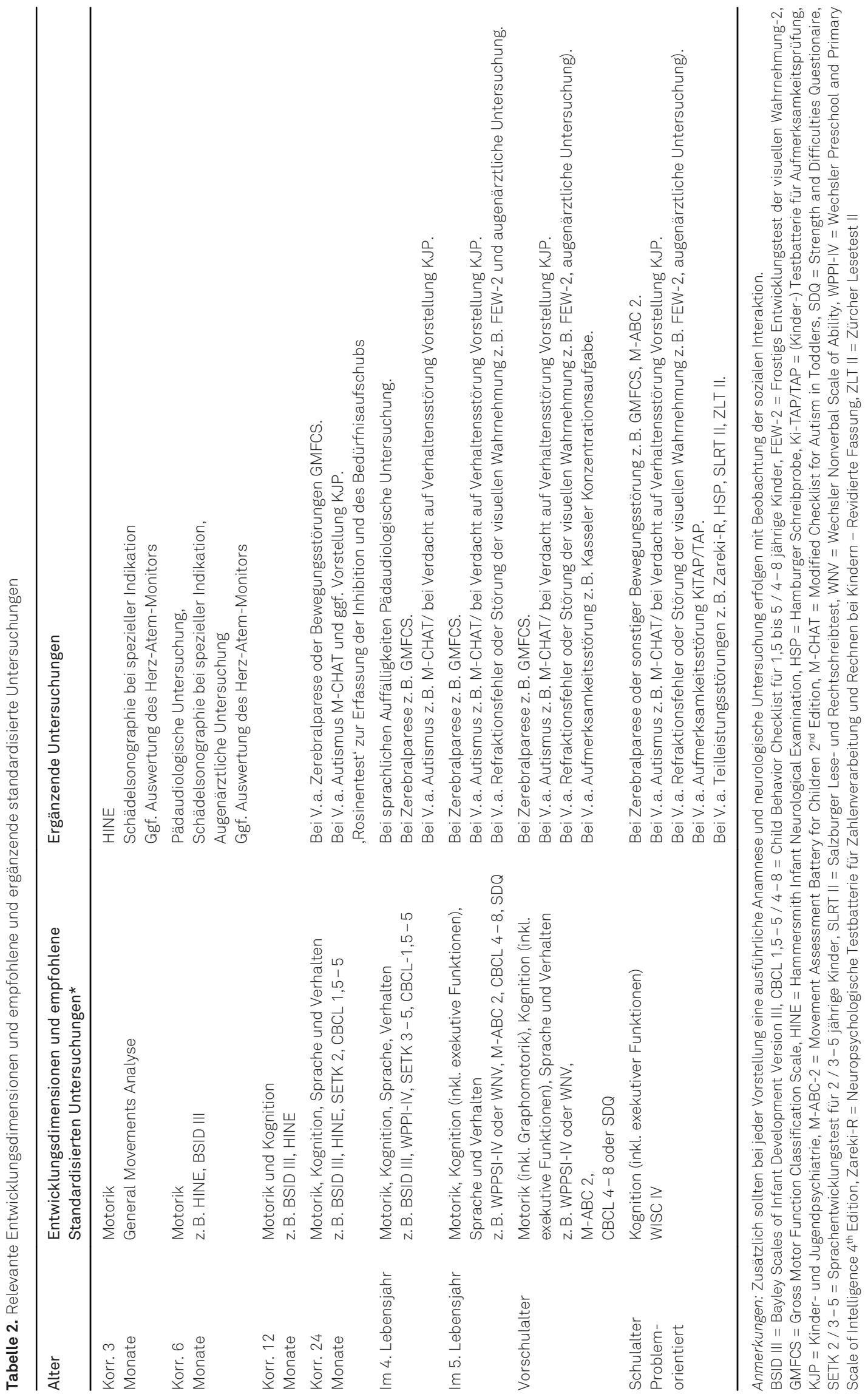


quotient (Blair et al., 2007; Blair et al., 2015; Hodel, 2018). Eine schlechte Impulskontrolle im Kleinkindalter zeigte sich zum Beispiel prädiktiv für Aufmerksamkeitsdefizite und geringere Schulleistungen im Alter von 8 Jahren (Jaekel, Eryigit-Madzwamuse et al., 2016). Da diese Defizite häufig erst dann bemerkt werden, wenn die Anforderungen an das Kind z. B. in der Schule steigen, ist eine frühere Identifikation und Förderung wünschenswert. Eine mögliche Intervention könnte hier das Training des zukunftsorientierten Denkens spielen. Frühgeborene zeigten zum Beispiel ein schlechteres Zeitverständnis im Alter von 4 Jahren als reifgeborene Kinder, und jene mit schlechtem Zeitverständnis besaßen ebenfalls eine geringere Impulskontrolle (Huening et al., 2017).

Testverfahren zu exekutiven Funktionen erscheinen daher im Vorschulalter sinnvoll, ebenso wie die Beurteilung der rezeptiven und expressiven Sprachfähigkeiten, und der fein- und graphomotorischen Entwicklung. Das Malen ist als Vorbereitung auf das Erlernen der Handschrift von besonderer Bedeutung. Graphomotorische Schwierigkeiten sollten im Vorschulalter bereits identifiziert werden, um vor der Einschulung mit einer Intervention zu beginnen und Folgen wie verkrampfte Stifthaltung und reduzierte Geschwindigkeit beim Schreiben vorzubeugen.

In einer kürzlich publizierten monozentrischen Studie mit 60 reifgeborenen Kindern und 60 sehr kleinen Frühgeborenen im Vorschulalter, die nach 2010 geboren worden waren und keine gravierenden Hirnschädigungen aufwiesen, wurde die graphomotorische Entwicklung untersucht (s. auch Abschnitt „Schulrückstellungen“). Dabei zeigen sehr Frühgeborene signifikant geringere Leistungen in der Graphomotorik, Feinmotorik und visuellräumlichen Wahrnehmung. 46.7\% der sehr Frühgeborenen haben eine Entwicklungsverzögerung der Graphomotorik, $45 \%$ der Feinmotorik und $23.3 \%$ der visuellräumlichen Wahrnehmung im Vergleich zu reifgeborenen Kindern im Vorschulalter (7\%, 12\%, 8\%) (Dathe et al., 2020).

\section{Schulreife und Schulerfolg bei Frühgeborenen}

Arbeitsgedächtnis- und Aufmerksamkeitsdefizite gehören zu den am besten dokumentierten Folgen einer zu frühen Geburt (Hall et al., 2012; Jaekel, Baumann et al., 2013; Jaekel, Wolke et al., 2013). Diese Defizite sind mit verantwortlich für den vielfach dokumentierten schulischen Misserfolg Frühgeborener (Mulder et al., 2010; Johnson, Wolke, et al., 2011; McBryde et al., 2020). Eltern und
Lehrer_innen von Frühgeborenen erwarten häufig, dass Entwicklungsrückstände bis zum Schuleintritt aufgeholt werden, das Gegenteil ist jedoch der Fall: kognitive Leistungen sind ab dem zweiten Lebensjahr relativ stabil, während gleichzeitig spezifische Lernstörungen erst in der Grundschule sichtbar werden, da differentielle Fähigkeiten stärker gefordert werden (Jaekel et al., 2011; Jaekel, Bartmann, et al., 2014; Jaekel \& Wolke, 2014; Breeman et al., 2015; Breeman et al., 2017).

Im Folgendem sollen Daten aus zwei unterschiedlichen eigenen Studien vorgestellt werden. In der ersten Untersuchung wurde die Schulrückstellungsquote anhand einer aktuellen Kohorte von Frühgeborenen im Vergleich zu reif geborenen Kindern im Vorschulalter ermittelt. Bei der zweiten Studie handelt es sich um eine Befragung von U.S.-amerikanischen Lehrer_innen mit dem Ziel, den Wissensstand zu langfristigen Folgen einer Frühgeburt, insbesondere Lernstörungen und Aufmerksamkeitsproblematik, aber auch die Möglichkeiten der schulischen Förderung zu erheben.

\section{Schulrückstellungen bei Frühgeborenen}

Internationale Studien haben in diesem Zusammenhang gezeigt, dass Frühgeborene ein erhöhtes Risiko für eine verspätete Schulreife haben (Roberts et al., 2011; Poehlmann, 2015).

Frühgeborene aus der Bayerischen Entwicklungsstudie wurden häufiger als Reifgeborene von der Einschulung zurückgestellt (Jaekel, Strauss, et al., 2015). Die Vor- und Nachteile einer Rückstellung von der Einschulung sind unter Bildungsexperten und Eltern Frühgeborener umstritten, da manche argumentieren, dass Kinder von der zusätzlichen Reifungszeit profitieren könnten (Odd et al., 2013; Fastenau, 2015). Quasi-experimentelle standardisierte Studienergebnisse zeigen jedoch, dass reines Aufschieben des Einschulungstermins, ohne spezifische Förderungsmaßnahmen oder Interventionen, die Lernfähigkeit und späteren Schulleistungen nicht unterstützt (Jaekel, Strauss et al., 2015). Aktuelle Daten einer an der Universitätsmedizin Essen durchgeführten Studie (Dathe et al., 2020) zeigen signifikante Unterschiede zwischen sehr Früh- und Reifgeborenen einer zwischen 2017 und 2019 eingeschulten Kohorte (siehe Tabelle 3 für deskriptive Charakteristika). In dem untersuchten Kollektiv war der Anteil der von der altersgemäßen Einschulung zurückgestellten Kinder bei den sehr frühgeborenen höher im Vergleich zu reifgeborenen Kontrollen. Während $12.5 \%$ der sehr Frühgeborenen von der regulären Ein- 
Tabelle 3. Merkmale der sehr früh- $(n=60)$ und reifgeborenen $(n=60)$ Teilnehmer zum Zeitpunkt der Geburt und bei Teilnahme an der Studie zur Graphomotorik im Vorschulalter (Dathe et al., 2020)

\begin{tabular}{llll}
\hline Merkmale & Sehr Frühgeborene & Reifgeborene & $p^{1}$ \\
\hline Weiblich, $n$ (\%) & $30(50)$ & $30(50)$ & 1.0 \\
Gestationsalter, W & $28,7(2,0)$ & $39,5(1,1)$ & $<.001$ \\
Geburtsgewicht, g & $1126(359)$ & $3414,3(494)$ & $<.001$ \\
<3. Perz., $n$ (\%) & $3(5)$ & $4(7)$ & .558 \\
Geburtslänge, cm & $36,7^{2}(4)$ & $51,7(3)$ & $<.001$ \\
Kopfumfang, cm & $26,3(3)$ & $35,1^{3}(1)$ & $<.001$ \\
Nabel-pH & $7,34^{4}(0,07)$ & $7,27^{5}(0,08)$ & $<.001$ \\
APGAR 5 min & $7,81(1,3)$ & $9,75(0,5)$ & $<.001$ \\
APGAR 10 min & $8,67(0,9)$ & $9,95(0,3)$ & $<.001$ \\
Mehrling, $n$ (\%) & $25(42)$ & $0(0)$ & $<.001$ \\
Alter, Jahre & $5,9(0,3)$ & $5,9(0,3)$ & .681 \\
Elterliche Bildung & & & .341 \\
Hoch, $n$ (\%) & $36(60)$ & $41(68)$ & $19(32)$ \\
Mittel, $n$ (\%) & $24(40)$ & & .029 \\
Schuleintritt ${ }^{6}$ & & $58(98,3)$ & $1(1,7)$ \\
Reguläre, $n$ (\%) & $49(87,5)$ & $7(12,5)$ & \\
Mit Rückstellung, $n$ (\%) & & \\
\hline
\end{tabular}

Anmerkungen: Die Daten werden als Mittelwerte mit (Standardabweichung) angegeben, wenn nicht explizit anders beschrieben. ${ }^{1}$ Es wurde ein t-Test für kontinuierliche und ein Chi-Quadrat-Test bzw. exakter Test nach Fisher für nicht kontinuierliche Daten durchgeführt. ${ }^{2} n=59 .{ }^{3} n=43 .{ }^{4} n=55 .{ }^{5} n=58 ;{ }^{6} n=$ 115 (FG $n=56$, RG $n=59$ ). $n=$ Anzahl; $p=$ Signifikanzwert; $W=$ Woche; $g=$ Gramm; Perz. = Perzentile; $c m=$ Zentimeter; min = Minute

schulung zurückgestellt wurden, waren dies nur $1.7 \%$ der reifgeborenen Kontrollkinder (Fisher Exact Probability Test $p=.029$; siehe auch Tab. 3).

Für die Stadt Essen im Bundesland Nordrhein-Westfalen berichtet das Gesundheitsamt im Schuljahr 2018/19 insgesamt 5160 Einschulungen, davon 129 Rückstellungen (2.5\%). In Anbetracht einer durchschnittlichen Frühgeborenenquote von $8.7 \%$ und einer angenommenen erhöhten Rückstellungsquote zwischen $2-12 \%$ in dieser Gruppe könnten entsprechend bis zu $42 \%$ der aktuell verspätet eingeschulten Kinder Frühgeborene sein.

Laut Bildungsbericht 2018 variiert die Schulrückstellungsquote aller Kinder, unabhängig vom Zeitpunkt ihrer Geburt, stark zwischen den einzelnen Bundesländern, nicht zuletzt wegen unterschiedlicher Regelungen des Stichtages der Einschulung (Autorengruppe Bildungsberichterstattung, 2018). In Bundesländern mit einem frühen Stichtag ist die Rate an Schulrückstellungen mit 14-20.5\% besonders hoch (Bayern, Brandenburg, Berlin). Im Gegensatz dazu liegt die Rückstellungquote in NRW, trotz vorgezogenem Stichtag nur bei $0.9 \%$, vermutlich deshalb, weil Schulrückstellungen nur „aus erheblichen gesundheitlichen Gründen" möglich sind. Somit weist die Stadt Essen eine deutlich höhere Quote auf, als der Landesdurchschnitt. Durch seine Lage im Ballungsraum Ruhrgebiet mit einem hohen Anteil von Kindern aus sozial schwachen oder bildungsfernen Familien, sowie einem hohen Anteil an Familien mit Migrationshintergrund könnten sich diese Zahlen erklären. Auf Grundlage zahlreicher Studien denken wir, dass die Effekte einer Frühgeburt, Migration, sowie sozialer Probleme stark miteinander korrelieren und sich schwer trennen lassen. Gleichzeitig diskutieren Forscher aktuell, in welchem Ausmaß diese kombinierten Effekte die erhöhte Schulrückstellungsquote entweder additiv oder multiplikatorisch beeinflussen. Leider können wir auf Grundlage der uns vorliegenden Daten diese Frage nicht beantworten.

\section{Bedeutung von Intervention und schulischer Förderung}

Grundsätzlich ermöglicht eine erfolgreiche frühe Intervention den größten Patientennutzen und erhöht das individuelle Potential zu gesellschaftlicher Teilhabe. Interventionen zur Förderung der kognitiven Entwicklung Frühgeborener durch erhöhte Stimulation wurden bisher meist im Säuglings- und Kleinkindalter durchgeführt. Diese zeigten jedoch wenig oder keine langfristigen Effekte (Orton et al., 2009). Aktuelle Studien belegen, dass das frühe Grundschulalter ein gutes Zeitfenster für Inter- 
Tabelle 4. Lehrerempfehlungen zur Förderung frühgeborener Kinder im Schulunterricht (unveröffentlichte Daten) (Elvert et al., 2020)

\begin{tabular}{|c|c|}
\hline Kodierkategorie & Kodiertes Inhaltsbeispiel \\
\hline \multicolumn{2}{|l|}{ Schulerfolg } \\
\hline Testung & Diagnostik, standardisierte Tests \\
\hline Anpassung von Aufgaben & Gruppenarbeit, Differenzierung von Aufgaben und Aktivitäten, Wiederholung von Instruktionen \\
\hline Anpassung der Klassenumgebung & Hilfestellungen, positive Atmosphäre im Klassenzimmer herstellen \\
\hline Gespräche initiieren & z.B. mit Kind, Eltern, Schulpsycholog_innen, etc. \\
\hline \multicolumn{2}{|l|}{ Aufmerksamkeit } \\
\hline Direkte Unterstützung & regelmäßige Erinnerungen und Kontrolle, Ablenkungen minimieren \\
\hline Anpassung von Aufgaben & Differenzierung von Aktivitäten, Aufgaben verkürzen \\
\hline Anpassung der Klassenumgebung & Partnerarbeit mit anderem Kind, Belohnungen \\
\hline Externe Unterstützung & z.B. Eltern, Kinderarzt/-ärztin, Schulpsycholog_innen, etc., involvieren \\
\hline \multicolumn{2}{|l|}{ Peerbeziehungen } \\
\hline Positive Gemeinschaft herstellen & Selbstvertrauen und Sozialverhalten fördern, positive Verstärkung \\
\hline Soziale Interaktion fördern & Gruppenarbeit, Gruppentische, Projektarbeit \\
\hline Externe Unterstützung & z.B. Sprachtherapeut_innen oder Schulpsycholog_innen involvieren \\
\hline \multicolumn{2}{|l|}{ Emotionen } \\
\hline Positive Umgebung herstellen & Selbstregulation fördern, Rückzugsmöglichkeiten bieten \\
\hline Anpassung von Arbeitsroutinen & Morgenkreis, Gruppenarbeit, regelmäßiger Austausch \\
\hline Gespräche initiieren & z.B. mit Kind, Eltern (Gründe identifizieren, Beziehungen aufbauen, ...) \\
\hline Externe Unterstützung & Verhaltensplan mit Hilfe vertrauter Personen erstellen \\
\hline
\end{tabular}

ventionen bei Frühgeborenen darstellen könnte (Jaekel et al., 2012; Wolke et al., 2013; Jaekel, Pluess, et al., 2015; Nichols et al., 2019; Jaekel et al., 2020). Ein Erklärungsansatz hierfür ist, dass Frühgeborene im Säuglingsalter alle zur Verfügung stehenden Ressourcen für erforderliche kortikale Reorganisation im Sinne funktionaler Adaptation verwenden, so dass kaum Potential übrigbleibt, von Stimulation durch die Umwelt zu profitieren. Im weiteren Entwicklungsverlauf dagegen etablieren sich funktionelle Strukturen und machen Kompensationseffekte möglich.

Ein zentraler Faktor für die Effektivität aller Fördermaßnahmen ist, dass diese adaptiv an die individuellen kindlichen Bedürfnisse angepasst werden (Jaekel et al., 2020). Bisher ist nicht klar, welche spezifischen Fördermaßnahmen Frühgeborenen langfristig helfen. Schulische Probleme in dieser Population gehen häufig mit Einschränkungen der Aufmerksamkeitsregulation und der Verarbeitungsgeschwindigkeit bei komplexen kognitiven Aufgaben einher (Mulder et al., 2010; Jaekel, Baumann et al., 2013; Jaekel, Wolke et al., 2013). Zudem können allgemeine kognitive Beeinträchtigungen, Sprachprobleme, Schwierigkeiten der sensorischen Verarbeitung und Probleme der Feinmotorik ein erfolgreiches Lernen erschweren.

\section{Lehrerbefragung zum Wissenstand über Folgen, spezifische Bedürfnisse und Förderung Frühgeborener}

In Bezug auf die spezifischen Bedürfnisse Frühgeborener im Schulunterricht liegen bisher nur limitierte Forschungsergebnisse und -empfehlungen vor. Onlinebefragungen von Lehrer_innen und Schulpsycholog_innen in Großbritannien und den USA haben gezeigt, dass Lehrkräfte vergleichsweise wenig über die langfristigen Folgen einer zu frühen Geburt wissen (Johnson et al., 2015). Gleichzeitig geben Lehrer_innen an, sich mehr Fortbildungsgelegenheiten in diesem Bereich zu wünschen (Elvert et al., 2020). Unser Team hat 246 Lehrer_innen aus den USA in einer triangulierten quantitativ/qualitativen Studie befragt, wie sie Frühgeborene im Unterricht in den Bereichen Schulerfolg, Aufmerksamkeit, Beziehungen zu Gleichaltrigen, und Emotionsregulation spezifisch fördern würden (Elvert et al. 2020). Die Antworten wurden mit thematischer Inhaltsanalyse ausgewertet (s. Tab. 4) und werden in zukünftigen Studien richtungsweisend sein für die Sammlung von Ideen zur Planung von Interventionen, zum Beispiel:

„Ich würde einen Teil der Arbeitsaufgaben reduzieren [...], Partnerarbeit fördern, [...] regelmäßig und in kurzen 
Abständen den Arbeitsstatus checken [...], und insgesamt sicherstellen, dass kleine Zwischenerfolge die Motivation aufrechterhalten“ (Teilnehmer \#84, übersetzt aus dem Englischen).

Obwohl die teilnehmenden Lehrkräfte dieser aktuellen US-Studie, wie schon zuvor für englische Lehrer_innen berichtet (Johnson et al., 2015), niedrig bis moderates Wissen über den langfristigen Einfluss von Frühgeburt auf Schulerfolg hatten, waren ihre Ideen und Vorschläge zur Förderung im Unterricht sehr sachkundig und konstruktiv. Insbesondere deckten die Vorschläge zur spezifischen Förderung FG im Schulunterricht die bekannten Entwicklungsdimensionen des Verhaltensphänotyps sehr gut ab (Konzentrations- und Aufmerksamkeitsprobleme, verlangsamte Verarbeitungsgeschwindigkeit, sozio-emotionale Schwierigkeiten). Dieser Befund unterstreicht, dass Fachwissen und Ausbildung zu FG im Schulalltag ggf. weniger wichtig sind als eine individuelle und umfassende Diagnostik. Jedes Kind besitzt ein individuelles Leistungsprofil, das mit seinen Stärken und Schwächen im Unterricht dementsprechend adaptiv gefördert werden muss. Eine neuere Studie zeigt, dass zum Beispiel Online-Angebote für Lehrer_innen eine gute Ressource darstellen, die den professionellen Wissensstand signifikant erhöhen kann (Johnson et al., 2019). Wir geben zu bedenken, dass Lehrer innen im Bereich der schulischen Förderung grundsätzlich die Hauptinterressengruppe mit der größten Expertise vertreten und unbedingt bei der Entwicklung und Planung von schulischen Interventionen und Fördermaßnahmen für Frühgeborene mit einbezogen werden müssen.

\section{Konklusion}

Ärzte/Ärztinnen, Psycholog_innen, Psychotherapeut_innen und Lehrer_innen sind in gleicher Weise gefordert, den besonderen Bedürfnissen Frühgeborener Rechnung zu tragen, um Spätfolgen zu minimieren und eine optimale Entfaltung ihres Potentials zu ermöglichen. Eine interdisziplinäre Zusammenarbeit der verschiedenen Berufs- und Interessengruppen, inklusive der Eltern und Frühgeborenen selbst, ist unverzichtbar (European Foundation for the care of newborn infants (EFCNI), 2018). Ebenso wäre es wünschenswert, dass neuere Befunde und Studienempfehlungen zeitnah von gesundheits- und bildungspolitischen Entscheidungsträgern berücksichtigt würden. Frühgeborene haben einen benachteiligten Start ins Leben, doch wenn wir es schaffen, ihre langfristige Entwicklung positiv zu begleiten und zu beeinflussen, können wir lebenslangen persönlichen und ökonomischen Erfolg und Lebensqualität fördern.

\section{Literatur}

Ancel, P. Y., Goffinet, F., EPIPAGE Writing Group, Kuhn, P., Langer, B., Matis, J. et al. (2015). Survival and morbidity of preterm children born at 22 through 34 weeks' gestation in France in 2011: Results of the EPIPAGE-2 cohort study. JAMA Pediatrics, 169, $230-238$.

Arbeitsgemeinschaft der Wissenschaftlichen Medizinischen Fachgesellschaften. [AWMF] (2018). Sozialpädiatrische Nachsorge extrem unreifer Frühgeborener mit einem Geburtsgewicht unter 1000 Gramm. Verfügbar unter: http://www.awmf.org/ leitlinien/detail/II/071-013.html

Autorengruppe Bildungsberichterstattung (2018). Nationaler Bildungsbericht. Verfügbar unter: https://www.bildungsbericht. de/de/bildungsberichte-seit-2006/bildungsbericht-2018/pdfbildungsbericht-2018/kapitel-c-2018.pdf

Bäuml, J. G., Daamen, M., Meng, C., Neitzel, J., Scheef, L., Jaekel, J. et al. (2014). Correspondence between Aberrant Intrinsic Network Connectivity and Gray-Matter Volume in the ventral brain of preterm born adults. Cerebral Cortex, 25, 4135-4145. https://doi.org/10.1093/cercor/bhu133.

Bilgin, A., Mendonca, M. \& Wolke, D. (2018). Preterm birth/low birth weight and markers reflective of wealth in adulthood: A meta-analysis. Pediatrics, 146, e20173625. https://doi.org/ 10.1542/peds.2017-3625

Bilgin, A. \& Wolke, D. (2016). Regulatory problems in very preterm and full-term infants over the first 18 months. Journal of Developmental and Behavioral Pediatrics, 37, 298-305.

Blair, C. \& Raver, C. C. (2015). School readiness and self-regulation: A developmental psychobiological approach. Annual Review of Psychology, 66, 711-731.

Blair, C. \& Razza, R. P. (2007). Relating effortful control, executive function, and false belief understanding to emerging math and literacy ability in kindergarten. Child Development, 78, $647-$ 663.

Bosanquet, M., Copeland, L., Ware, R. \& Boyd, R. (2013). A systematic review of tests to predict cerebral palsy in young children. Developmental Medicine and Child Neurology, 55, 418- 426.

Boyle, E. M. (2012). The late and moderate preterm baby. Seminars in Fetal \& Neonatal Medicine, 17, 119. https://doi.org/10.1016/ j.siny.2012.02.005

Breeman, L. D., Jaekel, J., Baumann, N., Bartmann, P. \& Wolke, D. (2015). Preterm cognitive function into adulthood. Pediatrics, 136, $415-423$.

Breeman, L. D., Jaekel, J., Baumann, N., Bartmann, P. \& Wolke, D. (2017). Neonatal predictors of cognitive ability in adults born very preterm: A prospective cohort study. Developmental Medicine and Child Neurology, 59, $477-483$.

Cheong, J. L. Y. \& Doyle, L. W. (2020). Long term outcomes in moderate and late preterm infants. In E. M. Boyle and J. Cusack (Eds.), Emerging Topics and Controversies in Neonatology (pp. 403 -413). Cham, CH: Springer Nature.

D’Onofrio, B. M., Class, Q. A., Rickert, M. E., Larsson, H., Langstrom, N. \& Lichtenstein, P. (2013). Preterm birth and mortality and morbidity: A population-based quasi-experimental study. JAMA Psychiatry, 70, $1231-1240$.

Darlow, B. A., Horwood, L. J., Pere-Bracken, H. M. \& Woodward, L. J. (2013). Psychosocial outcomes of young adults born very low birth weight. Pediatrics, 132, e1521-1528.

Dathe, A., Jaekel, J., Felderhoff-Mueser, U. \& Huening, B. (2020). Visual perception, fine motor and visual motor skills in very preterm and term born children before school entry. Unpublished Manuscript, University Duisburg-Essen.

DeMaster, D., Bick, J., Johnson, U., Montroy, J. J., Landry, S. \& Duncan, A. F. (2019). Nurturing the preterm infant brain: Le- 
veraging neuroplasticity to improve neurobehavioral outcomes. Pediatric Research, 85, $166-175$.

Einspieler, C., Bos, A. F., Krieber-Tomantschger, M., Alvarado, E., Barbosa, V. M., Bertoncelli, N. et al. (2019). Cerebral palsy: Early markers of clinical phenotype and functional outcome. Clinical Medicine, 8, 1616. https://doi.org/10.3390/jcm8101616

Einspieler, C., Prechtl., H., Bos, A. F., Ferrari, F. \& Cioni, G. (2004). Prechtel's method on the qualitative assessment of general movements in preterm, term and young infants. Cambridge, UK: Mac Keith Press.

Elvert, C., Johnson, S. \& Jaekel, J. (2020). Teachers' knowledge and approaches towards supporting preterm children in the classroom. Unpublished Manuscript, University of Tennessee.

European Foundation for the care of newborn infants (EFCNI). (2018). European standards of care for newborn health. Retrieved from https://newborn-health-standards.org/standards/followupcontinuing-care/overview/

Fastenau, P. S. (2015). Are the data on delayed school entry compelling enough to change policy ... or even pediatrician recommendations? Developmental Medicine and Child Neurology, 57, $596-597$.

Feldman, R. (2015). The adaptive human parental brain: implications for children's social development. Trends in Neurosciences, 38, $387-399$.

Feldman, R., Granat, A., Pariente, C., Kanety, H., Kuint, J. \& GilboaSchechtman, E. (2009). Maternal depression and anxiety across the postpartum year and infant social engagement, fear regulation, and stress reactivity. Journal of the American Academy of Child and Adolescent Psychiatry, 48, 919-927.

G-BA (2017). Richtlinie des G-BA (Gemeinsamer Bundesausschuss) über Maßnahmen zur Qualitätssicherung der Versorgung von Früh- und Reifgeborenen gemäß §136 Abs. 1 Nr. 2 SGB V in Verbindung mit §92 Abs. 1 Satz 2 Nr. 13 SGB V (Qualitätssicherungs-Richtlinie Früh- und Reifgeborene/QFR-RL). Verfügbar unter https://www.g-ba.de/downloads/62-4921446/QFR-RL_2017-06-15_iK-2017-08-25.pdf

Greenough, A. (2013). Long-term respiratory consequences of premature birth at less than 32 weeks of gestation. Early Human Development, 89 (Suppl 2), S25-27.

Hack, M. (2009). Adult outcomes of preterm children. Journal of Developmental and Behavioral Pediatrics, 30, 460-470.

Hall, J. \& Wolke, D. (2012). A comparison of prematurity and small for gestational age as risk factors for age $6-13$ year emotional problems. Early Human Development, 88, 797-804.

Heinonen, K., Eriksson, J. G., Kajantie, E., Pesonen, A. K., Barker, D. J., Osmond, C. et al. (2013). Late-preterm birth and lifetime socioeconomic attainments: The Helsinki birth cohort study. Pediatrics, 132, $647-655$.

Helenius, K., Sjors, G., Shah, P. S., Modi, N., Reichman, B., Morisaki, N. et al. (2017). Survival in very preterm infants: An international comparison of 10 national neonatal networks. Pediatrics, 140, e20171264. https://doi.org/10.1542/peds.20171264

Herting, E., Kribs, A., Hartel, C., von der Wense, A., Weller, U., Hoehn, T. et al. (2020). Two-year outcome data suggest that less invasive surfactant administration (LISA) is safe. Results from the follow-up of the randomized controlled AMV (avoid mechanical ventilation) study. European Journal of Pediatrics, 179, $1309-1313$

Heuser, K. M., Jaekel, J. \& Wolke, D. (2018). Origins and predictors of friendships in 6- to 8-year-old children born at neonatal risk. The Journal of Pediatrics, 193, 93-101 (e105).

Hirvonen, M., Ojala, R., Korhonen, P., Haataja, P., Eriksson, K., Gissler, M. et al. (2018). Visual and hearing impairments after preterm birth. Pediatrics, 146, e20173888. https://doi.org/ 10.1542/peds.2017-3888
Hodel, A. S. (2018). Rapid infant prefrontal cortex development and sensitivity to early environmental experience. Developmental Review, 48, $113-144$.

Hovi, P., Vohr, B., Ment, L. R., Doyle, L. W., McGarvey, L., Morrison, K. M. et al. (2016). Blood pressure in young adults born at very low birth weight: Adults born preterm international collaboration. Hypertension, 68, 880-887.

Huening, B. M., Assing, B., Weishaupt, E., Dransfeld, F., FelderhoffMuser, U. \& Zmyj, N. (2017). Delay of gratification and time comprehension is impaired in very preterm children at the age of 4 years. Early Human Development, 115, 77-81.

Huening, B. M., Haertel, C., Wolke, D., Wassernaer-Leemhuis, A. \& EFCNI (2018). European Standards of Care for Newborn Health: Transition from hospital to home. Retrived from https://newborn-health-standards.org/transition-hospital-home/

Huening, B. M., Reimann, M., Beerenberg, U., Stein, A., Schmidt, A. \& Felderhoff-Muser, U. (2012). Establishment of a family-centred care programme with follow-up home visits: Implications for clinical care and economic characteristics. Klinische Pädiatrie, 224, 431- 436 .

Humberg, A., Spiegler, J., Fortmann, M. I., Zemlin, M., Marissen, J., Swoboda, I. et al. (2020). Surgical necrotizing enterocolitis but not spontaneous intestinal perforation is associated with adverse neurological outcome at school age. Scientific Reports, 10, 2373.

Institut für Qualitätssicherung und Transparenz im Gesundheitswesen (IQTIG). (2019). Qualitätsreport 2019. Verfügbar unter: https://iqtig.org/downloads/berichte/IQTIC_Qualitaetsreport2019_2019-09-25.pdf

Jaekel, J. (2016). Commentary: Supporting preterm children's parents matters - A reflection on Treyvaud et al. (2016). Journal of Child Psychology and Psychiatry, 57, 822 - 823.

Jaekel, J. (2020). Commentary: The impact of preterm birth on the life course: Assessment challenges and future directions - A reflection on Darlow et al. 2020. Developmental Medicine and Child Neurology, 62, 1347-1347. https://doi.org/10.1111/ dmcn.14692

Jaekel, J., Bartmann, P., Schneider, W. \& Wolke, D. (2014). Neurodevelopmental pathways to preterm children's specific and general mathematic abilities. Early Human Development, 90, $639-644$

Jaekel, J., Baumann, N., Bartmann, P. \& Wolke, D. (2018). Mood and anxiety disorders in very preterm/very low-birth weight individuals from 6 to 26 years. Journal of Child Psychology and Psychiatry, 59, $88-95$.

Jaekel, J., Baumann, N., Bartmann, P. \& Wolke, D. (2019). General cognitive but not mathematic abilities predict very preterm and healthy term born adults' wealth. PLoS One, 14, e0212789.

Jaekel, J., Baumann, N. \& Wolke, D. (2013). Effects of gestational age at birth on cognitive performance: A function of cognitive workload demands. PLoS One, 8, e65219.

Jaekel, J., Eryigit-Madzwamuse, S. \& Wolke, D. (2016). Preterm toddlers' inhibitory control abilities predict attention regulation and academic achievement at age 8 years. The Journal of Pediatrics, 169, 87 - 92 e81.

Jaekel, J., Heuser, K. M., Zapf, A., Roll, C., Brevis Nunez, F., Bartmann, P. et al. (2020). Preterm children's long-term academic performance after adaptive computerized training: An efficacy and process analysis of a randomised controlled trial. Pediatric Research, https://doi.org/10.1038/s41390-020-01114-w

Jaekel, J., Pluess, M., Belsky, J. \& Wolke, D. (2015). Effects of maternal sensitivity on low birth weight children's academic achievement: A test of differential susceptibility versus diathesis stress. Journal of Child Psychology and Psychiatry, 56, 693 701. 
Jaekel, J., Sorg, C., Baeuml, J., Bartmann, P. \& Wolke, D. (2019). Head growth and intelligence from birth to adulthood in very preterm and term born Individuals. Journal of the International Neuropsychological Society, 25, 48-56.

Jaekel, J., Strauss, V. Y., Johnson, S., Gilmore, C. \& Wolke, D. (2015). Delayed school entry and academic performance: A natural experiment. Developmental Medicine and Child Neurology, 57, 652 - 659. https://doi.org/10.1111/dmcn.12713

Jaekel, J. \& Wolke, D. (2011). Teacher rated attention problems predict academic achievement in very preterm and fullterm adolescents. In J. Özyurt, A. Anschütz, S. Bernholt and J. Lenk (Eds.), Interdisciplinary perspectives on cognition, education and the brain (pp. 195-202). Oldenburg: BIS-Verlag der Carl von Ossietzky Universität Oldenburg.

Jaekel, J. \& Wolke, D. (2014). Preterm birth and dyscalculia. The Journal of Pediatrics, 164, 1327-1332.

Jaekel, J., Wolke, D. \& Bartmann, P. (2013). Poor attention rather than hyperactivity/impulsivity predicts academic achievement in very preterm and full-term adolescents. Psychological Medicine, 43, $183-196$.

Jaekel, J., Wolke, D. \& Chernova, J. (2012). Mother and child behaviour in very preterm and term dyads at 6 and 8 years. Developmental Medicine and Child Neurology, 54, 716-723.

Johnson, S., Bamber, D., Bountziouka, V., Clayton, S., Cragg, L., Gilmore, C. et al. (2019). Improving developmental and educational support for children born preterm: Evaluation of an elearning resource for education professionals. BMJ Open, 9, e029720.

Johnson, S., Gilmore, C., Gallimore, I., Jaekel, J. \& Wolke, D. (2015). The long-term consequences of preterm birth: What do teachers know? Developmental Medicine and Child Neurology, 57, 571 - 577. https://doi.org/10.1111/dmcn.12683

Johnson, S. \& Marlow, N. (2011). Preterm birth and childhood psychiatric disorders. Pediatric Research, 69, 11R-18R.

Johnson, S. \& Marlow, N. (2014). Growing up after extremely preterm birth: Lifespan mental health outcomes. Seminars in Fetal \& Neonatal Medicine, 19, 97-104.

Johnson, S., Strauss, V., Gilmore, C., Jaekel, J., Marlow, N. \& Wolke, D. (2016). Learning disabilities among extremely preterm children without neurosensory impairment: Comorbidity, neuropsychological profiles and scholastic outcomes. Early Human Development, 103, 69-75.

Johnson, S., Wolke, D., Hennessy, E. \& Marlow, N. (2011). Educational outcomes in extremely preterm children: Neuropsychological correlates and predictors of attainment. Developmental Neuropsychology, 36, 74-95.

Kajantie, E., S. Johnson, K. Heinonen, P. J. Anderson, D. Wolke, K. A. I. Evensen, K. et al. (2020). Common Core Assessments in follow-up studies of adults born preterm-Recommendation of the Adults Born Preterm International Collaboration. Paediatric and Perinatal Epidemiology, https://doi.org/10.1111/ppe.12694

Khan, K. A., Petrou, S., Dritsaki, M., Johnson, S. J., Manktelow, B., Draper, E. S. et al. (2015). Economic costs associated with moderate and late preterm birth: A prospective population-based study. BJOG, 122, $1495-1505$.

Korvenranta, E., Lehtonen, L., Peltola, M., Hakkinen, U., Andersson, S., Gissler, M. et al. (2009). Morbidities and hospital resource use during the first 3 years of life among very preterm infants. Pediatrics, 124, $128-134$.

Kwong, A. K. L., Fitzgerald, T. L., Doyle, L. W., Cheong, J. L. Y. \& Spittle, A. J. (2018). Predictive validity of spontaneous early infant movement for later cerebral palsy: A systematic review. Developmental Medicine and Child Neurology, 60, 480-489.

Lenzen-Schulte, M. (2018). Frühgeburt als Syndrom: Kranke Lunge, schwächelnder IQ. Deutsches Ärzteblatt, 115, A-648/ B-561 /C-562.
Linsell, L., Johnson, S., Wolke, D., O’Reilly, H., Morris, J. K., Kurinczuk, J. J. et al. (2018). Cognitive trajectories from infancy to early adulthood following birth before 26 weeks of gestation: A prospective, population-based cohort study. Archives of Disease in Childhood, 103, $363-370$.

Lipkind, H. S., Slopen, M. E., Pfeiffer, M. R. \& McVeigh, K. H. (2012). School-age outcomes of late preterm infants in New York City. American Journal of Obstetrics \& Gynecology, 206, 222 e221226.

Lohaugen, G. C., Gramstad, A., Evensen, K. A., Martinussen, M., Lindqvist, S., Indredavik, M. et al. (2010). Cognitive profile in young adults born preterm at very low birthweight. Developmental Medicine and Child Neurology, 52, 1133-1138.

Lovering, A. T., Elliott, J. E., Laurie, S. S., Beasley, K. M., Gust, C. E., Mangum, T. S. et al. (2014). Ventilatory and sensory responses in adult survivors of preterm birth and bronchopulmonary dysplasia with reduced exercise capacity. Annals of the American Thoracic Society, 11, 1528-1537.

MacKay, D. F., Smith, G. C., Dobbie, R. \& Pell, J. P. (2010). Gestational age at delivery and special educational need: Retrospective cohort study of 407,503 schoolchildren. PLoS Med, 7, e1000289.

Markopoulou, P., Papanikolaou, E., Analytis, A., Zoumakis, E. \& Siahanidou, T. (2019). Preterm birth as a risk factor for metabolic syndrome and cardiovascular disease in adult life: A systematic review and meta-analysis. The Journal of Pediatrics, 210, $69-80$ e65.

Marlow, N., Wolke, D., Bracewell, M. A. \& Samara, M. (2005). Neurologic and developmental disability at six years of age after extremely preterm birth. The New England Journal of Medicine, 352, $9-19$.

McBryde, M., Fitzallen, G. C., Liley, H. G., Taylor, H. G. \& Bora, S. (2020). Academic outcomes of school-aged children born preterm: A systematic review and meta-analysis. JAMA Network Open, 3, e202027.

Mendonca, M., Bilgin, A. \& Wolke, D. (2019). Association of preterm birth and low birth weight with romantic partnership, sexual intercourse, and parenthood in adulthood: A systematic review and meta-analysis. JAMA Network Open, 2, e196961.

Moore, T., Hennessy, E. M., Myles, J., Johnson, S. J., Draper, E. S., Costeloe, K. L. et al. (2012). Neurological and developmental outcome in extremely preterm children born in England in 1995 and 2006: The EPICure studies. BMJ, 345, e7961.

Morrison, K. M., Ramsingh, L., Gunn, E., Streiner, D., Van Lieshout, R., Boyle, M. et al. (2016). Cardiometabolic health in adults born premature with extremely low birth weight. Pediatrics, 138, e20160515. https://doi.org/10.1542/peds.2016-0515

Moster, D., Lie, R. T. \& Markestad, T. (2008). Long-term medical and social consequences of preterm birth. The New England Journal of Medicine, 359, $262-273$.

Mulder, H., Pitchford, N. J. \& Marlow, N. (2010). Processing speed and working memory underlie academic attainment in very preterm children. Archives of Disease in Childhood. Fetal and Neonatal Edition, 95, F267-272.

Nichols, T., Jaekel, J., Bartmann, P. \& Wolke, D. (2019). Differential susceptibility effects of maternal sensitivity in childhood on small for gestational age adults' wealth. Development and Psychopathology, 32, 197-203. https://doi.org/10.1017/ S0954579418001669

Nosarti, C., Murray, R. \& Hack, M. (2010). Neurodevelopmental outcome of preterm birth. Cambridge: Cambridge University Press.

Odd, D., Evans, D. \& Emond, A. (2013). Preterm birth, age at school entry and educational performance. PLoS One, 8, e76615.

Orton, J., Spittle, A., Doyle, L., Anderson, P. \& Boyd, R. (2009). Do early intervention programmes improve cognitive and motor 
outcomes for preterm infants after discharge? A systematic review. Developmental Medicine and Child Neurology, 51, $851-$ 859.

Pace, C. C., Spittle, A. J., Molesworth, C. M., Lee, K. J., Northam, E. A., Cheong, J. L. et al. (2016). Evolution of depression and anxiety symptoms in parents of very preterm infants during the newborn period. JAMA Pediatrics, 170, 863-870.

Parkinson, J. R., Hyde, M. J., Gale, C., Santhakumaran, S. \& Modi, N. (2013). Preterm birth and the metabolic syndrome in adult life: A systematic review and meta-analysis. Pediatrics, 131, e1240-1263.

Patel, R. M. (2016). Short- and long-term outcomes for extremely preterm infants. American Journal of Perinatology, 33, 318328.

Peterson, B. S. (2003). Brain imaging studies of the anatomical and functional consequences of preterm birth for human brain development. Annals of the New York Academy of Sciences, 1008, $219-237$.

Petrou, S., Abangma, G., Johnson, S., Wolke, D. \& Marlow, N. (2009). Costs and health utilities associated with extremely preterm birth: Evidence from the EPICure study. Value Health, 12, 1124-1134.

Pierrat, V., Marchand-Martin, L., Arnaud, C., Kaminski, M., RescheRigon, M., Lebeaux, C. et al. (2017). Neurodevelopmental outcome at 2 years for preterm children born at 22 to 34 weeks' gestation in France in 2011: EPIPAGE-2 cohort study. BMJ, 358, j3448.

Pimperton, H., Blythe, H., Kreppner, J., Mahon, M., Peacock, J. L., Stevenson, J. et al. (2016). The impact of universal newborn hearing screening on long-term literacy outcomes: A prospective cohort study. Archives of Disease in Childhood, 101, 9-15.

Platt, M. J. (2014). Outcomes in preterm infants. Public Health, $128,399-403$.

Pluess, M. (2015). Individual differences in environmental sensitivity. Child Development Perspectives, 9, 138-143.

Poehlmann-Tynan, J., Gerstein, E. D., Burnson, C., Weymouth, L., Bolt, D. M., Maleck, S. \& Schwichtenberg A. J. (2015). Risk and resilience in preterm children at age 6. Development and Psychopathology, 27, 843-858.

Poulsen, G., Wolke, D., Kurinczuk, J. J., Boyle, E. M., Field, D., Alfirevic, Z. et al. (2013). Gestational age and cognitive ability in early childhood: A population-based cohort study. Paediatric and Perinatal Epidemiology, 27, $371-379$.

Quigley, M. A., Poulsen, G., Boyle, E., Wolke, D., Field, D., Alfirevic, Z. et al. (2012). Early term and late preterm birth are associated with poorer school performance at age 5 years: A cohort study. Archives of Disease in Childhood - Fetal and Neonatal Edition, 97, F167-173.

Raju, T. N. K., Pemberton, V. L., Saigal, S., Blaisdell, C. J., MoxeyMims, M., Buist, S. et al. (2017). Long-term healthcare outcomes of preterm birth: An executive summary of a conference sponsored by the National Institutes of Health. The Journal of Pediatrics, 181, $309-318$ e301.

Reyes, L. M., Jaekel, J., Heuser, K. M. \& Wolke, D. (2019). Developmental cascades of social inhibition and friendships in preterm and full-term children. Infant and Child Development, 28, e2165.

Reyes, M. L., Jaekel, J. \& Wolke, D. (2019). Effects of gestational age and early parenting on children's social inhibition at 6 years. Children, 6(7), 81. https://doi.org/10.3390/children6070081

Roberts, G., Lim, J., Doyle, L. W. \& Anderson, P. J. (2011). High rates of school readiness difficulties at 5 years of age in very preterm infants compared with term controls. Journal of Developmental and Behavioral Pediatrics, 32, 117-124.

Rogers, C. E., Lean, R. E., Wheelock, M. D. \& Smyser, C. D. (2018). Aberrant structural and functional connectivity and neurode- velopmental impairment in preterm children. Journal of Neurodevelopmental Disorders, 10, 38.

Saigal, S., Day, K. L., Van Lieshout, R. J., Schmidt, L. A., Morrison, K. M. \& Boyle, M. H. (2016). Health, wealth, social integration, and sexuality of extremely low-birth-weight prematurely born adults in the fourth decade of life. JAMA Pediatrics, 170, 678686.

Santhakumaran, S., Statnikov, Y., Gray, D., Battersby, C., Ashby, D., Modi, N. et al. (2018). Survival of very preterm infants admitted to neonatal care in England 2008-2014: Time trends and regional variation. Archives of Disease in Childhood. Fetal and Neonatal Edition, 103, F208-F215.

Serenius, F., Ewald, U., Farooqi, A., Fellman, V., Hafstrom, M., Hellgren, K. et al. (2016). Neurodevelopmental outcomes among extremely preterm infants 6.5 years after active perinatal care in Sweden. JAMA Pediatrics, 170, 954-963.

Shonkoff, J. P. \& Fisher, P. A. (2013). Rethinking evidence-based practice and two-generation programs to create the future of early childhood policy. Development and Psychopathology, 25, $1635-1653$.

Singer, D. (2006). Die Langzeitprognose von Frühgeborenen: Was sich hinter den Statistiken verbirgt. Zeitschrift für Geburtshilfe und Neonatologie, 210, 50-59.

Spittle, A., Orton, J., Anderson, P. J., Boyd, R. \& Doyle, L. W. (2015). Early developmental intervention programmes provided post hospital discharge to prevent motor and cognitive impairment in preterm infants. Cochrane Database Syst Rev, 11, CD005495. https://doi.org/10.1002/14651858.CD005495.pub4

St Clair, M. C., Pickles, A., Durkin, K. \& Conti-Ramsden, G. (2011). A longitudinal study of behavioral, emotional and social difficulties in individuals with a history of specific language impairment (SLI). Journal of Communication Disorders, 44, 186-199.

Stenson, C., Wood, C. \& Embleton, N. (2016). Catch-up growth and the developmental origins of health in preterm infants. Infant, $12,82-86$.

Stoll, B. J., Hansen, N. I., Bell, E. F., Walsh, M. C., Carlo, W. A., Shankaran, S. et al. (2015). Trends in care practices, morbidity, and mortality of extremely preterm neonates, 1993-2012. JAMA, 314, $1039-1051$.

Tran, L., Huening, B. M., Kaiser, O., Schweiger, B., Sirin, S., Quick, H. H. et al. (2017). Cerebellar-dependent associative learning is impaired in very preterm born children and young adults. Scientific Reports, 7, 18028.

Twilhaar, E. S., de Kieviet, J. F., Aarnoudse-Moens, C. S., van Elburg, R. M. \& Oosterlaan, J. (2018). Academic performance of children born preterm: A meta-analysis and meta-regression. Archives of Disease in Childhood: Fetal \& Neonatal, 103, F322F330.

van Bel, F., Vaes, J. \& Groenendaal, F. (2019). Prevention, reduction and repair of brain injury of the preterm infant. Frontiers in Physiology, 10:181. https://doi.org/10.3389/fphys.2019.00181

van Haastert, I. C., Groenendaal, F., Uiterwaal, C. S., Termote, J. U., van der Heide-Jalving, M., Eijsermans, M. J. et al. (2011). Decreasing incidence and severity of cerebral palsy in prematurely born children. The Journal of Pediatrics, 159, 86-91.e81.

van Noort-van der Spek, I. L., Franken, M. C. \& Weisglas-Kuperus, N. (2012). Language functions in preterm-born children: a systematic review and meta-analysis. Pediatrics, 129, $745-754$.

Vigod, S. N., Villegas, L., Dennis, C. L. \& Ross, L. E. (2010). Prevalence and risk factors for postpartum depression among women with preterm and low-birth-weight infants: A systematic review. BJOG, 117, $540-550$.

Volpe, J. J. (2009). Brain injury in premature infants: A complex amalgam of destructive and developmental disturbances. Lancet Neurol, 8, 110-124. 
Volpe, J. J. (2011). Systemic inflammation, oligodendroglial maturation, and the encephalopathy of prematurity. Annals of Neurology, 70, $525-529$

Wolke, D., Baumann, N., Busch, B. \& Bartmann, P. (2017). Very preterm birth and parents' quality of life 27 years later. Pediatrics, 140, e20171263. https://doi.org/10.1542/peds.20171263

Wolke, D., Baumann, N., Strauss, V., Johnson, S. \& Marlow, N. (2015). Bullying of preterm children and emotional problems at school age: Cross-culturally invariant effects. The Journal of Pediatrics, 166, $1417-1422$.

Wolke, D., Jaekel, J., Hall, J. \& Baumann, N. (2013). Effects of sensitive parenting on the academic resilience of very preterm and very low birth weight adolescents. Journal of Adolescent Health, 53, 642-647.

Wolke, D., Johnson, S. \& Mendonça, M. (2019). The life course consequences of very preterm birth. Annual Review of Developmental Psychology, 1, 69-92.

Woodward, L. J., Anderson, P. J., Austin, N. C., Howard, K. \& Inder, T. E. (2006). Neonatal MRI to predict neurodevelopmental out- comes in preterm infants. The New England Journal of Medicine, 355, $685-694$.

Woodward, L. J., Clark, C. A., Bora, S. \& Inder, T. E. (2012). Neonatal white matter abnormalities an important predictor of neurocognitive outcome for very preterm children. PLoS One, 7, e51879. Woythaler, M. (2019). Neurodevelopmental outcomes of the late preterm infant. Seminars in Fetal and Neonatal Medicine, 24, $54-59$.

\section{PD Dr. Britta Maria Hüning}

Klinik für Kinderheilkunde I, Neonatologie

Pädiatrische Intensivmedizin und Neuropädiatrie

Universitätsklinikum Essen

Universität Duisburg-Essen

Hufelandstraße 55

45122 Essen

Britta.Huening@uk-essen.de 